\title{
Wireless World Research Forum (WWRF)
}

\section{Feasibility of Providing High Data Rate Coverage in Cellular Fixed Relay Networks}

\author{
Hakan Bolukbasi ${ }^{*}$, Halim Yanikomeroglu*, David D. Falconer*, Shalini Periyalwar** \\ *Broadband Communications \& Wireless Systems (BCWS) Centre, \\ Dept. of Systems and Computer Engineering, Carleton University, Ottawa, Canada \\ ${ }^{* *}$ Nortel Networks, Ottawa, Canada \\ e-mail: \{hakan,halim,ddf\}@sce.carleton.ca, speriyal@nortelnetworks.com
}

\begin{abstract}
In this contribution the feasibility of providing high data rate coverage through fixed relay stations (RSs) in cellular networks is studied, under the (somewhat conservative) constraint that all the hops in a composite multi-hop link use orthogonal channels. Spectral efficiency is used as the performance metric. It is shown that, high data rate coverage is obtained by relatively high number of relays; on the contrary high number of hops is not required since that results in loss of available channels.
\end{abstract}

Index Terms-Relaying, Mesh Networks, Multi-hop Networks.

\section{INTRODUCTION}

EUTURE wireless systems are expected to provide very high data rates; it is estimated that, for $4 G$ systems, the aggregate peak data rate demands for mobile access and nomadic/local area wireless access could be around 100 Mbps and 1 Gbps, respectively [1]. With the existing cellular architecture, this goal cannot be realized over a wide coverage area. Therefore, some novel cellular network architectures are being studied.

Towards that end, multi-hop relaying concept is considered to be a promising solution [2]. Adding relays in the cells reduces the signal transmission distances, resulting in lower propagation loss and higher average SNR to the mobile user. Therefore, range extension and outage reduction can be obtained by deploying relays [3]. In addition, relays are much simpler devices than base stations in the sense that they require low transmit power and limited functionality [4]; that is, their complexity is comparable to that of WLAN access points. In fact relays are more cost effective due to the fact that, unlike WLAN access points, they do not require a wired backhaul connection.

In this contribution the feasibility of providing high data rate coverage through fixed relay stations (RSs) in cellular networks is studied, under the (somewhat conservative) constraint that all the hops in a composite multi-hop link use orthogonal channels. Spectral efficiency is used as the performance metric. The main idea behind this is spectral efficiency is directly proportional to the data rate. Therefore, maximum throughput is obtained if all users in the system can be provided by the maximum available spectral efficiency. It is shown that, high data rate coverage is obtained by relatively high number of relays; on the contrary high number of hops is not required since that results in loss of available channels.

\section{System Model}

In a cellular network topology, connections between nodes can be established as either single-hop or multi-hop links. In this context, a hop is considered as the direct wireless connection (or link) between two nodes. This implies that, for a multi-hop communication between two nodes, some number of intermediate nodes are needed to relay the data to be sent from source node to destination node. Fig 1 shows the nodes and links for the multi-hop communication between the Central Node $(\mathrm{CN})$ and the 


\section{Wireless World Research Forum (WWRF)}

Mobile Station (MS). It is worth mentioning that the figure shows the topology, not the exact positions, of the nodes; they don't have to be positioned on a linear structure. In the figure, "square" node represents the $\mathrm{CN}$, equivalent to a base station in Conventional Cellular Networks (CCN), and has a connection to the wired network. The "triangle" node represents the MS and each "circle" node represents a Relay Station (RS). All the links between CN, RSs, and MSs are wireless. Spectral efficiency values (in $\mathrm{bits} / \mathrm{sec} / \mathrm{Hz}$ ) for multi-hop links that connects $\mathrm{CN}$ to MS are denoted by $a_{1}$ to $a_{n}$. Similarly, spectral efficiency of the single-hop link between the $\mathrm{CN}$ and the same MS is denoted by $b$. Each spectral efficiency is determined from the signal-to-interference-plus-noise ratio (SINR) of the corresponding link. Although the issue of signalling overhead is not considered in this contribution, its effect can be assumed to be included in the spectral efficiency.

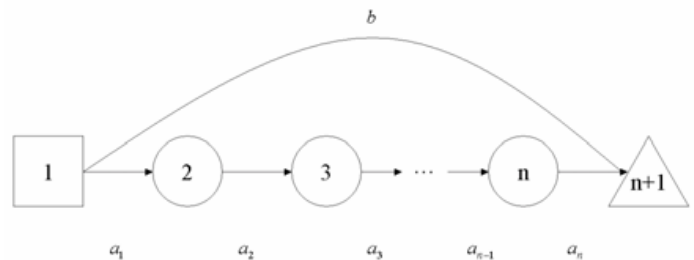

Fig. 1. Topology of nodes and links for the communication between $\mathrm{CN}$ and MS.

For each RS in the system, the amount of data received and the amount of data transmitted must be equal:

$$
\operatorname{Data}(I N)=\operatorname{Data}(O U T) \text {. }
$$

The total amount of data (in terms of bits), received or transmitted, can be expressed as follows:

$$
\begin{aligned}
\text { Data } & =\text { SpectralEfficiency }(\mathrm{bits} / \mathrm{Hz} / \mathrm{sec}) \\
& \times \text { Time }(\mathrm{sec}) \times \text { Bandwidth }(\mathrm{Hz}) .
\end{aligned}
$$

Then, from (1) \& (2),

$$
\left(S E_{I N}\right)\left(T_{I N}\right)\left(B_{I N}\right)=\left(S E_{\text {OUT }}\right)\left(T_{\text {OUT }}\right)\left(B_{\text {OUT }}\right),
$$

where $S E$ stands for spectral efficiency, $T$ for time, and $B$ for bandwidth.
Assuming that the same amount of bandwidth is used for both receiving and transmitting links,

$$
a_{1} T_{1}=a_{2} T_{2}=a_{3} T_{3}=\ldots=a_{n} T_{n}=b T .
$$

At this point it is clear that the total transmission time for single-hop communication is $T$, since there is only one link available. However this is not the case for multi-hop communication; each multi-hop link uses some amount of time for the transmission, i.e. $i$ th link uses $T_{i}$, and total transmission time for multi-hop communication is the 'union' of all time 'set's used for the link, considering some of the sets may intersect with the others:

Total transmission time for multi-hop communication: $T_{1} \cup T_{2} \cup T_{3} \cup \ldots \cup T_{n}$

Total transmission time for single-hop communication: $T$

It is aimed that the RSs would be simple and small devices compared to base stations, so they would need two separate channels for downlink and uplink. In this contribution, it is assumed a relay does not transmit and receive at the same time. It is also assumed that all other time 'set's for the composite multi-hop link are orthogonal to reduce the interference in the cell. Then,

Total transmission time for multi-hop communication: $T_{1}+T_{2}+T_{3}+\ldots+T_{n}$

Total transmission time for single-hop communication: $T$

\section{Comparison of the Data Rates}

Data rate can be thought as the amount of data (information) that can be transferred per unit time. Therefore to find the data rate for either communication types, i.e. multi-hop or single-hop, the total amount of data must be divided by the total amount of time and the ratio of multi-hop data rate to single-hop data can be found as follows:

$$
\frac{\text { Data Rate }_{\mathrm{MH}}}{\text { Data Rate }_{\mathrm{SH}}}=\frac{\text { Data }_{M H} / T_{M H}}{\operatorname{Data}_{S H} / T_{S H}},
$$




\section{Wireless World Research Forum (WWRF)}

where

Data $_{M H}$ : Total amount of data for the Multi-hop transmission

$T_{M H}$ : Total amount of time for the Multihop transmission

Data $_{S H}$ : Total amount of data for the Single-hop transmission

$T_{S H} \quad$ : Total amount of time for the Singlehop transmission.

For the same user, to find the ratio of the data rates for multi-hop and single-hop cases in a fair manner, either total amount of data or total amount of time for both multihop and single-hop transmissions should be taken as equal. Without loss of generality, total amount of data is assumed to be equal for both cases:

$\frac{\text { Data Rate }_{\mathrm{MH}}}{\text { Data Rate }_{\mathrm{SH}}}=\frac{1 / T_{M H}}{1 / T_{S H}}=\frac{T_{S H}}{T_{M H}}$.

Then,

$$
\begin{aligned}
\frac{\text { Data Rate }_{\mathrm{MH}}}{\text { Data Rate }_{\mathrm{SH}}} & =\frac{T}{T_{1}+T_{2}+T_{3}+\ldots+T_{n}} \\
& =\frac{\frac{T}{T_{1}}}{\frac{T_{1}}{T_{1}}+\frac{T_{2}}{T_{1}}+\frac{T_{3}}{T_{1}}+\ldots+\frac{T_{n}}{T_{1}}} .
\end{aligned}
$$

From (4),

$$
\begin{aligned}
\frac{\text { Data Rate }_{\mathrm{MH}}}{\text { Data Rate }_{\mathrm{SH}}} & =\frac{\frac{a_{1}}{b}}{\frac{a_{1}}{a_{1}}+\frac{a_{1}}{a_{2}}+\frac{a_{1}}{a_{3}}+\ldots+\frac{a_{1}}{a_{n}}} \\
& =\frac{\frac{1}{b}}{\frac{1}{a_{1}}+\frac{1}{a_{2}}+\frac{1}{a_{3}}+\ldots+\frac{1}{a_{n}}} \\
& =\frac{\frac{1}{a_{1}}+\frac{1}{a_{2}}+\frac{1}{a_{3}}+\ldots+\frac{1}{a_{n}}}{b} .
\end{aligned}
$$

In (8), single-hop data rate seems like proportional to the single-hop spectral efficiency, namely $b$, and multi-hop data rate is proportional to the result of some arithmetical operations of the spectral efficiency value of multi-hop links. Therefore, to make it similar to the single-hop case, the result can be thought as 'combined spectral efficiency':

$\frac{\text { Data Rate }_{\mathrm{MH}}}{\text { Data Rate }_{\mathrm{SH}}}=\frac{a_{c}}{b}$,

where $a_{c}$ is defined as combined n-hop spectral efficiency and $a_{c}=\frac{1}{\sum_{i=1}^{n} \frac{1}{a_{i}}}$.

For multi-hop communication to be superior over single-hop communication, multi-hop data rate must be higher than single-hop data rate. Therefore, following constraint must be satisfied:

$\frac{\text { Data Rate }_{\mathrm{MH}}}{\text { Data Rate }_{\mathrm{SH}}} \geq 1 \Rightarrow b \leq a_{c}$,

In order to find the closest distance to the $\mathrm{CN}$ where multi-hop is better than single-hop in terms of data rate, best performance of multi-hop communication should be obtained, which requires all the links of multihop communication to have the best spectral efficiency available, i.e., $a_{i}=a_{\max }$. This distance indicates that no matter how good the multi-hop links are, even for the maximum spectral efficiency values, only the users beyond this distance should be assisted via multi-hop so that they have higher data rates than the case they use single-hop communication.

At this point, it should be noticed that if all the links have same spectral efficiency value, then the best performance for multi-hop is achieved by 2-hop communication since the combined spectral efficiency is maximum for the smallest value of number of hops, namely $n$, for such a case. Therefore, the closest point to the $\mathrm{CN}$ where multi-hop communication is superior to single-hop 


\section{Wireless World Research Forum (WWRF)}

communication must be through 2-hop communication.

For 2-hop communication with the links having spectral efficiency of $a_{\max }$, (10) becomes $b \leq a_{\max } / 2$. That is, for the usage of 2-hop communication, the threshold combined spectral efficiency value is $a_{\max } / 2$. In other words, if the spectral efficiency for single-hop communication is greater than or equal to $a_{\max } / 2$, multi-hop communication, even for the best case: 2hop communication where both links have spectral efficiency of $a_{\max }$ which results a combined spectral efficiency of $a_{\max } / 2$, cannot improve the data rate of the transmission; so it's better to use single-hop communication for users having single-hop spectral efficiency greater than $a_{\max } / 2$. On the other hand, if the spectral efficiency for single-hop communication is less than $a_{\max } / 2$, best case of 2-hop communication becomes superior over single-hop communication. Therefore, 2-hop communication is enabled in that region if combined spectral efficiency of multi-hop links, i.e. CN-RS \& RS-MS links, is greater than the spectral efficiency of the single-hop link, i.e. CN-MS link, for the same user.

For 3-hop communication with all the links having spectral efficiency of $a_{\max }$, the threshold combined spectral efficiency becomes $a_{\max } / 3$. In other words, if the single-hop spectral efficiency is greater than $a_{\max } / 3$, even if all the links of 3-hop communication has maximum available spectral efficiency, single-hop communication performs better than 3-hop communication. In general, if single-hop spectral efficiency is greater than $a_{\max } / n$, even the best $n$-hop communication cannot outperform single-hop communication. Therefore, to determine the closest point where $n$-hop communication can be used, spectral efficiency for the single-hop communication should be known. Figure 2 shows the spectral efficiency coverage circles for the maximum spectral efficiency value of $6 \mathrm{bits} / \mathrm{sec} / \mathrm{Hz}$.

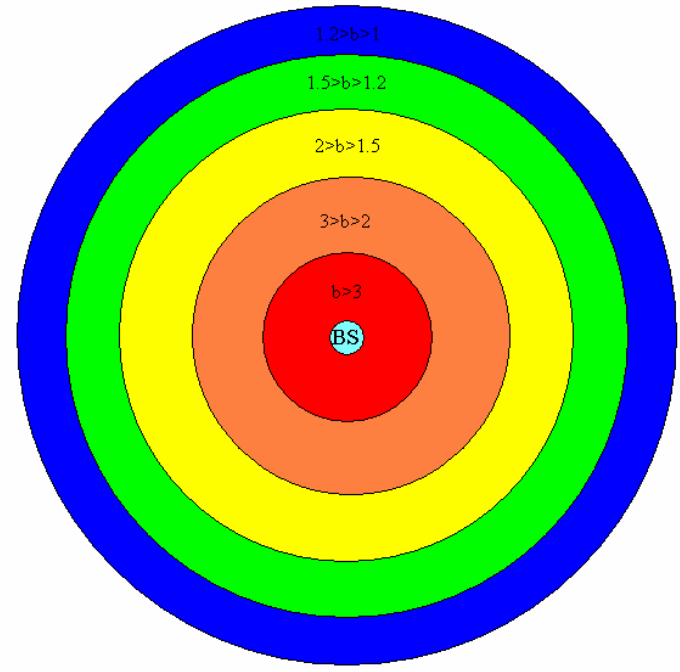

Fig. 2. Spectral Efficiency circles for $a_{\max }=6$

- $\quad$ In Fig 2, red region, i.e. area of the inner most circle, represents the region where single-hop spectral efficiency is greater than 3 ; in that region none of the multihop cases can perform better than the single-hop case, so, users in that region should use the direct link with the $\mathrm{CN}$.

- In the orange region, the spectral efficiency of single-hop communication is between 2 and 3, so in that region 3hop communication cannot perform better than single-hop communication, then users in that region should use single-hop or 2-hop depending on the combined spectral efficiency values.

- In the yellow region, the spectral efficiency of single-hop communication is between 1.5 and 2, so in that region 4hop communication cannot perform better than single-hop communication, then users in that region should use single-hop, 2-hop, or 3-hop depending on the combined spectral efficiency values.

- In the green region, the spectral efficiency of single-hop communication is between 1.2 and 1.5 , so in that region 5-hop communication cannot perform better than single-hop communication, then users in that region should use single-hop, 2-hop, 3-hop or 4-hop depending on the combined spectral efficiency values, and so on. 


\section{Wireless World Research Forum (WWRF)}

\section{Estimation of Radii for Spectral Efficiency Coverage Circles}

As the starting point, instead of multi-hop only 2-hop case is considered. Cell area is divided into two regions, $\mathrm{R} 1$ and $\mathrm{R} 2$, as shown in Fig 3 . The radius of the inner circle is $r_{1}$ and the radius for the outer one is $R$. Users in R1 get spectral efficiency greater than or equal to $a_{\max } / 2$ from the BS. Therefore, for those users single-hop communication is superior to any kind of multi-hop communication. On the other hand, in R2, depending on the combined spectral efficiency, multi-hop communication may be superior to sing-hop one. The ultimate aim, in this case, is to provide the maximum spectral efficiency available to the users in R2, which is $a_{\max } / 2$, via multi-hop.

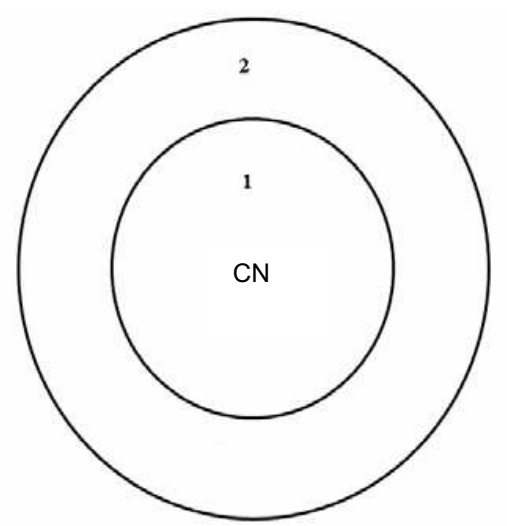

Fig. 3. Region 1 \& Region 2 for the cell

The radii of the circles in Fig 3 are determined by the spectral efficiency values. Fig 4 shows the graph of one-one correspondence of SINR and the spectral efficiency values.

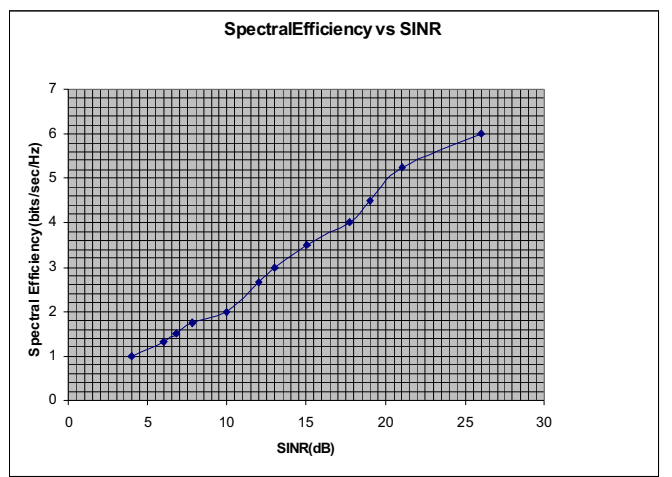

Fig 4. Spectral Efficiency vs. SINR Graph.
SINR can be written as follows:

$$
\gamma^{d B}=10 \log \frac{S}{I+N} \Rightarrow S=(I+N) * 10^{\frac{\gamma^{d B}}{10}},
$$

where $\gamma^{d B}$ is SINR in $\mathrm{dB}, S$ is the received signal power, $I$ is the interference power and $N$ is the noise power. The received signal power can be written as follows (shadowing effect is ignored):

$S=C \frac{P_{C N}}{r^{n}}$,

where $P_{C N}$ is the transmit power of the $\mathrm{CN}$, $r$ is the transmission distance, $C$ is a constant, and $n$ is the propagation constant. Then,

$$
\begin{aligned}
C \frac{P_{C N}}{r^{n}} & =(I+N) 10^{\frac{\gamma^{d B}}{10}} \\
\Rightarrow r^{n} & =C \frac{P_{C N}}{(I+N) 10^{\frac{\gamma^{d B}}{10}}} .
\end{aligned}
$$

At this point, to simplify the analysis, some assumptions are made:

- Noise power in (12) is assumed to be uniform throughout the cell; in other words, all the users are faced with the same amount of noise power.

- The system is assumed to be noise limited since with the use of relays, the coverage area of the cells may be larger than the conventional cell areas and some portion of the users use lowpowered relay assistance, reducing the interference for the neighbor cells.

Therefore, (12) can be modified as follows:

$$
r^{n}=C \frac{P_{C N}}{N 10^{\frac{\gamma^{d B}}{10}}} \text {. }
$$

Equation (13) can be used to find the radius of inner circle in Fig 3 . As mentioned before, $r_{1}$ corresponds to the radius of the region where spectral efficiency is greater than or equal to $a_{\text {max }} / 2 . \ln$ Fig $4, a_{\max }$ is 6 $\mathrm{bits} / \mathrm{sec} / \mathrm{Hz}$, so $a_{\max } / 2$ corresponds to 3 


\section{Wireless World Research Forum (WWRF)}

bits $/ \mathrm{sec} / \mathrm{Hz}$, which requires SINR value of 13 $\mathrm{dB}$ for $16-\mathrm{QAM}$ rate $(3 / 4)$. Then,

$$
r_{1}^{n}=C \frac{P_{C N}}{N 10^{\frac{13}{10}}} .
$$

Let's assume that the coverage are of the cell is where spectral efficiency is greater than 1 or SINR greater than 4 (for QPSK rate $(1 / 2))$. Then,

$$
\begin{aligned}
& R^{n}=\frac{P_{C N}}{N 10^{\frac{4}{10}}} \\
& \Rightarrow \frac{R}{r_{1}}=10^{\frac{9}{10^{* n}}} .
\end{aligned}
$$

\section{Estimation of Relay Positions}

It is assumed that relays are positioned on a circle forming tiers around the $\mathrm{CN}$. In order to find the position of the $1^{\text {st }}$ tier relays, consider a user in R2 but just on the border of R1. This user can get a spectral efficiency of almost $a_{\max } / 2$ from the $\mathrm{CN}$ using singlehop. If multi-hop is to be used for the communication of this user, same spectral efficiency should be provided. So, if the nearest RS is located at a distance of $(r+d)$ from the $\mathrm{CN}$, that RS should provide spectral efficiency of $a_{\max }$ at the distance $d$, just on the border of R1. Therefore, that user would be provided with the maximum combined spectral efficiency for multi-hop communications, which is $a_{\max } / 2$.

Similar to, previously done radius finding calculations, $d$ can be found as follows:

$$
\begin{gathered}
d^{n}=\frac{P_{R S}}{(I+N) 10^{\frac{\gamma_{1}^{d B}}{10}}} \\
r_{1}^{n}=\frac{P_{C N}}{(I+N) 10^{\frac{\gamma_{2}^{d B}}{10}}},
\end{gathered}
$$

where $\gamma_{1}^{d B}$ is the SINR value corresponding to the maximum spectral efficiency, $a_{\max }$, and $\gamma_{2}^{d B}$ is the SINR value corresponding to half of the maximum spectral efficiency, $a_{\max } / 2$. From the two equations,

$\left(\frac{d}{r_{1}}\right)^{n}=\frac{P_{R S}}{P_{C N}} 10^{\frac{\gamma_{2}^{d B}-\gamma_{1}^{d B}}{10}}$.

For $a_{\max }=6, \gamma_{1}^{d B}$ and $\gamma_{2}^{d B}$ values are $26 \mathrm{~dB}$ and $13 \mathrm{~dB}$, respectively. Then, (17) becomes:

$\left(\frac{d}{r_{1}}\right)^{n}=\frac{P_{R S}}{P_{C N}} 10^{-1.3}$.

For different values of the propagation constant $n$ and the ratio $P_{R S} / P_{C N}$, the radius $d$ can be found in terms of $r_{1}$. Table 1

\begin{tabular}{|c|c|c|}
\hline \multicolumn{3}{|c|}{ ANALYTICAL RESULTS } \\
\hline n & 4 & 3.5 \\
\hline $\mathrm{d} / \mathrm{r} 1$ for $\mathrm{Prs} / \mathrm{Pbs}=0.1$ & 0.26 & 0.22 \\
\hline$(2 \mathrm{~d}+\mathrm{r} 1) / \mathrm{r} 1$ for $\mathrm{Prs} / \mathrm{Pbs}=0.1$ & 1.53 & 1.44 \\
\hline rcell/r1 & 1.67 & 1.80 \\
\hline SINR by the relay at Cell Border & 18.3 & 11.0 \\
\hline SE of the relay at the Cell Border & 4.2 & 2.3 \\
\hline CSE of relay at Cell Border & 2.47 & 1.66 \\
\hline Number of Relays needed & 17 & 20 \\
\hline $\mathrm{d} / \mathrm{r} 1$ for $\mathrm{Prs} / \mathrm{Pbs}=0.2$ & 0.31 & 0.26 \\
\hline$(2 \mathrm{~d}+\mathrm{r} 1) / \mathrm{r} 1$ for $\mathrm{Prs} / \mathrm{Pbs}=0.2$ & 1.63 & 1.53 \\
\hline rcell/r1 & 1.67 & 1.80 \\
\hline SINR by the relay at Cell Border & 23.6 & 15.3 \\
\hline SE of the relay at the Cell Border & 5.75 & 3.6 \\
\hline CSE of relay at Cell Border & 2.94 & 2.25 \\
\hline Number of Relays needed & 15 & 17 \\
\hline
\end{tabular}
shows all the values to find the optimum relay numbers and positions.

TABLE I

ANALYTICAL RESULTS

Following definitions are used in the table: d/r1: Ratio of Relay Station coverage radius to inner circle radius.

$(2 \mathrm{~d}+\mathrm{r} 1) / \mathrm{r} 1$ : Maximum distance from $\mathrm{CN}$ that the users are provided with spectral efficiency greater than (for single-hop) or equal (for 2-hop) to 3.

R/r1: Ratio of cell radius to innermost circle radius.

SINR by the relay at the cell border: The SINR value for the link between RS and a MS positioned at the cell border. 


\section{Wireless World Research Forum (WWRF)}

SE by the relay at the cell border: Spectral Efficiency of link between RS and a MS positioned at the cell border.

CSE of the relay at Cell Border: Combined Spectral Efficiency of 2-hop communication for a MS positioned at the cell border.

Fig 5 \& 6 shows the cell dimensions and RS positions according to the values from the table for propagation constant values of 4 and 3.5. As the reference dimension, the value of $r_{1}$, radius of the circle in which the $\mathrm{CN}$ serves spectral efficiency of $a_{\max } / 2$, is taken as 1 unit and according to this value the cell radius and RS coverage area circle radius values are found. Fig 5 is for propagation constant of 4 . For this scheme several values of RS power results in different coverage areas. As seen from the figure, if the RS power is 0.2 times the $\mathrm{CN}$ power, the area between two circles can almost be covered with relay stations of one tier. If we assume that the distance between two RS is $\sqrt{3}$ times the radius of its coverage area and all the RSs are deployed on a circle, total number of RS required can be found as 15 . On the other hand, as seen from the Figure 6 , for $n=3.5$ case, with the same power, 0.2 times the power of the BS, only half of the R2 is covered by the RS with the maximum spectral efficiency. If the power of the RS is not increased, then, there should be more than one tier of RSs in R2.

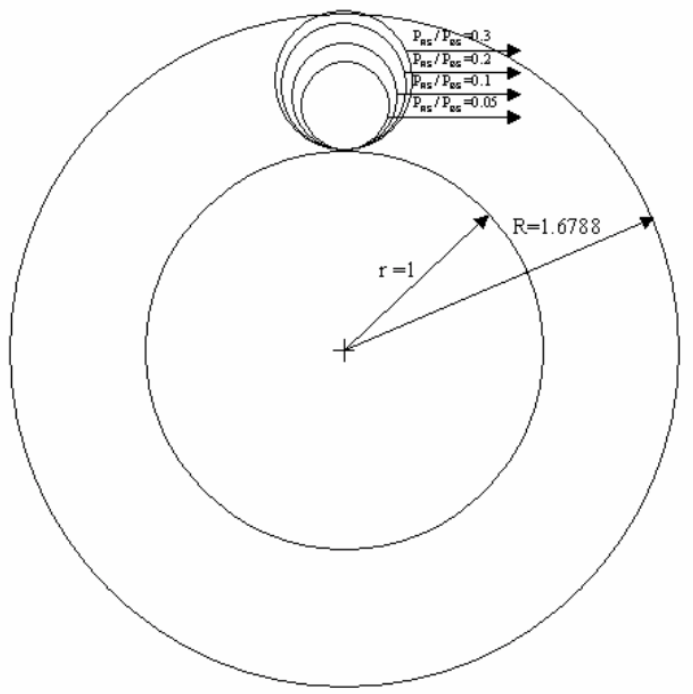

Fig. 5. Cell structure for $n=4$.

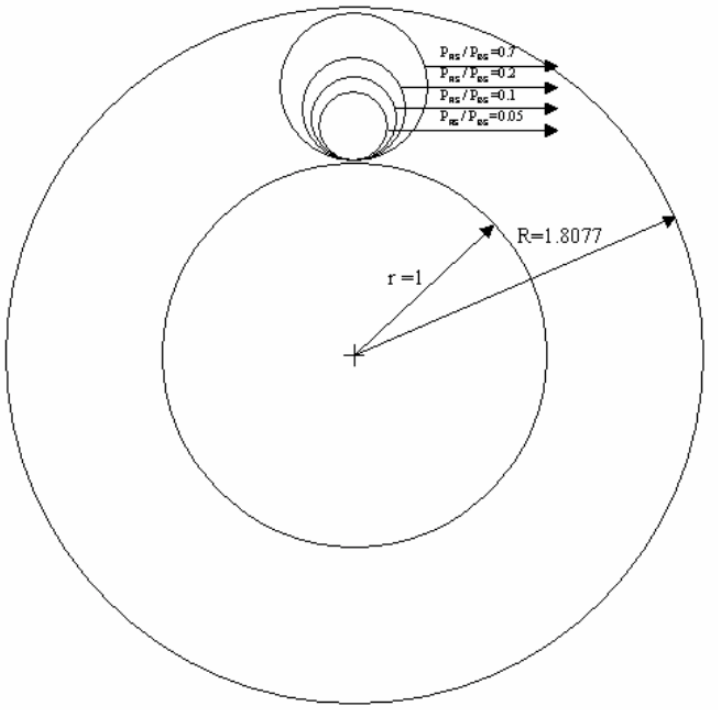

Fig. 6 . Cell structure for $n=3.5$.

\section{Conclusion}

In this contribution the feasibility of providing high data rate coverage through fixed relay stations (RSs) in cellular networks is studied, under the (somewhat conservative) constraint that all the hops in a composite multihop link use orthogonal channels. It is demonstrated that, if the RS positions are chosen properly, "ubiquitous" high data rate coverage can indeed be provided without consuming additional bandwidth --- but with a relatively high number of RSs. It is shown, on the other hand, that the required number of hops is quite modest; two tiers of RSs around each $\mathrm{CN}$, enabling at most 3-hops, would be sufficient. If the ubiquity condition is relaxed (by allowing small regions, especially close to the cell border, to have slightly less data rates), then in most circumstances only one tier of RSs (i.e., maximum two hops) will be sufficient to provide the required coverage.

\section{REFERENCES}

[1] W. Mohr, R. Luder and K.-H Mohrmann, "Data rate estimates, range calculations and spectrum demand for new elements of systems beyond IMT2000," $5^{\text {th }}$ International Symposium on Wireless Personal Multimedia Communications (WPMC), vol. 1, pp. 37-46, 2002.

[2] H. Yanikomeroglu, "Fixed and mobile relaying technologies for cellular networks," 2nd Workshop on Applications and Services in Wireless Networks (ASWN), pp. 75-81, 2002. 


\section{Wireless World Research Forum \\ (WWRF)}

[3] Huining $\mathrm{Hu}$, Halim Yanikomeroglu, David D. Falconer, and Shalini Periyalwar, "Range extension without capacity penalty in cellular networks with digital fixed relays," accepted to IEEE Globecom'04.

[4] Ralf Pabst, Bernhard H. Walke, Daniel C. Schultz, Patrick Herhold, Halim Yanikomeroglu, Sayandev Mukherjee, Harish Viswanathan, Matthias Lott, Wolfgang Zirwas, Mischa Dohler, Hamid Aghvami, David D. Falconer, and Gerhald P. Fettweis, "Relay-based deployment concepts for wireless and mobile broadband cellular radio", IEEE Communications Magazine, vol. 42, no. 9, pp. 8089, September 2004. 